\title{
Modeling of the Twin-Roll Casting Process: Transition from Casting to Rolling
}

\author{
Christian Rodrigues $^{1}$ (D) Andreas Ludwig ${ }^{1} \cdot$ Abdellah Kharicha $^{1} \cdot$ Menghuai Wu $^{1}$
}

Received: 17 July 2018/Accepted: 6 September 2018/Published online: 29 September 2018

(C) The Author(s) 2018

\begin{abstract}
During twin-roll casting, an alloy melt is passing the gap between two counter-rotating rolls, where cooling and solidification leads to the continuous formation of a solid strand. In order to describe this process, a two-phase Eulerian-Eulerian volume-averaging model is presented that accounts for (1) transport and growth of spherical grains within a flowing melt, (2) the formation of a coherent solid network above a specific solid fraction and (3) the viscoplastic flow of the solid network with the interstitial melt during casting and compression. For the considered case of an inoculated $\mathrm{Al}-4 \mathrm{wt} \% \mathrm{Cu}$ alloy, the process conditions are chosen such that two relatively thick viscoplastic semi-solid shells meet between the rolls, and thus, the material is pressed together and squeezed against the casting direction. The squeezed out material consists of segregated melt and some solid that quickly disappears after melting. It is observed during this study that macrosegregation distributions are inherently connected to the mush deformation that is enforced during the hot rolling process.
\end{abstract}

Keywords Twin-roll casting · Solidification · Macrosegregation · Viscoplasticity · Two-phase flow

Christian Rodrigues

christian.gomes-rodrigues@unileoben.ac.at

1 Department Metallurgy, Montanuniversitaet Leoben, FranzJosef-Str. 18, 8700 Leoben, Austria

\section{Introduction}

Twin-roll continuous casting is an emerging technology in the casting industry. The process consists of introducing the melt directly into the gap between two counter-rotating rolls, which are cooled so that a solidifying shell forms on each of the moving roll surfaces. Due to the progressively reducing dimensions of the gap between the two rolls, the two shells meet at the so-called kissing point, creating a continuous solidified strip, which is a close-to-final product. The reduced number of operation steps compared to conventional technologies is one of the primary advantages of this technology. As a result, also the operating and energy requirement costs are typically lower $[1,2]$.

The main feature of twin-roll casting is that, both casting and rolling are merged into one single continuous step. Therefore, a thermo-fluid-mechanical model must be applied for the numerical simulation of this process. Often this is done by using a single phase finite element code originally designed for pure rolling, and treating the liquid as a solid with low viscosity [3, 4]. If a liquid core still exists at the roll nip, the mechanical part of the simulation can be omitted and only solidification and flow should be modeled [5]. However, the viscoplasticity of the semi-solid slurry must explicitly be treated if solidification has already reached the strand center before passing the roll nip. According to Nguyen et al. [6], the solid-liquid mixture can be considered as a viscoplastic continuous solid skeleton saturated with interstitial liquid. Therefore, solid and liquid phases become inherently coupled: if on the one hand, pressing the solid skeleton drives the fluid flow behavior, on the other, the resulting pressure distribution in the interstitial liquid affects the equivalent stress experienced by the solid phase. 
In the present paper, a two-phase Eulerian-Eulerian volume-averaging approach is presented to model the transport and growth of equiaxed crystals during solidification. Furthermore, in order to account for mush deformation, a solution algorithm has been developed to include a viscoplastic model when the concentration of solid is above a certain criterion. The model is implemented in OpenFOAM and tested in a pertinent industrial application, where the significance of the viscoplastic behavior of the solid phase cannot be neglected.

\section{Model Description}

The simulation of the twin-roll continuous casting presented in this paper relies on a two-phase Eulerian-Eulerian volume-averaging model. The general approach solves mass, momentum, species and enthalpy conservation equations for each phase. Additionally, a transport equation is also taken into account for the calculation of the number density of grains across the domain. A detailed description of the corresponding governing equations has been made in previous publications [7, 8], so the interested reader can refer to those contributions. For the sake of conciseness, here only the most relevant equations will be highlighted.

The general governing equations are shown in Table 1, where $\rho$ denotes the density, $\mathbf{v}$ the velocity vector field, $\tau$ the deviatoric stress tensor, $p$ the pressure, $c$ the solute concentration, $h$ the enthalpy per unit mass, $\mathbf{q}$ the heat flow vector, $n$ the grain number density, and the subscripts $i$ refer to either liquid $(\ell)$ or solid $(s)$ phase. For $i=\ell$, the minus sign from ' $\mp$ ' must be taken, whereas for $i=s$ the plus sign must be taken. The sum of both liquid and solid fraction is equal to one. Also, it is worth noting that the effect of gravity has been neglected in the current work.

The critical step in the present model is to define the deviatoric stress term in the momentum equations, which for the liquid phase can be written as:

$\tau_{\ell}=2 \mu_{\ell} \dot{\varepsilon}_{\ell}$

where the strain-rate tensor is given by $2 \dot{\varepsilon}_{\ell}=\nabla \mathbf{v}_{\ell}+$ $\left(\nabla \mathbf{v}_{\ell}\right)^{T}$ and the liquid viscosity is assumed to be constant $\left(\mu_{\ell}=0.013 \mathrm{~Pa} \cdot \mathrm{s}\right)$. As for the effective solid deviatoric stress tensor, it can be divided into two regimes which depend on the amount of solid in the region under consideration as:

$\tau_{s}^{\text {eff }}= \begin{cases}2 \mu_{s} \dot{\varepsilon}_{s} & \text { for } g_{s} \leq g_{s}^{t} \\ \mu_{s}^{\text {app }}\left(\frac{2}{A} \dot{\varepsilon}_{s}-\left(\frac{2}{3 A}-\frac{1}{9 B}\right) \operatorname{tr}\left(\dot{\varepsilon}_{s}\right) \mathbf{I}\right) & \text { for } g_{s}>g_{s}^{t}\end{cases}$

In Eq. 7, $g_{s}^{t}$ determines the onset of the viscoplastic regime. Based on the investigation of the mechanical behavior of an A356 alloy in the semi-solid state, Nguyen et al. [6] suggested that the viscoplastic behavior appears at a solid fraction of $57 \%$.

Specifically for $g_{s}>g_{s}^{t}$, we enter into the viscoplastic regime and the solid skeleton should be expressed with an incompressible viscoplastic Norton-Hoff model [9]. Here, $A$ and $B$ are rheological parameters which, according to Nguyen et al. [6], depend upon the solid fraction as

$A=\frac{3}{g_{s}^{6.47}} \quad$ and $\quad B=\frac{1}{g_{s}^{6.94}}-1$.

Another important distinction between the two solid phase regimes has to do with how the viscosity is calculated. For dilute and very dilute flows, Ishii [10] postulated that the mixture viscosity of a set of particles can be expressed according to the power-law viscosity model. Considering the mixing rule for the viscosity, the corresponding solid viscosity is given as:

$\mu_{s}=\frac{\mu_{\ell}}{g_{s}}\left(\left(1-\frac{g_{s}}{g_{s}^{p}}\right)^{-2.5 g_{s}^{p}}-\left(1-g_{s}\right)\right)$

where $g_{s}^{p}$ is the random packing limit (which can be identified as either the loose or close one). According to Olmedilla et al. [11], $g_{s}^{p}$ is found to occur at a volume fraction of $58.5 \%$.

For the viscoplastic regime, the corresponding apparent viscosity is given by

$\mu_{s}^{\mathrm{app}}=3 K_{v}\left(\sqrt{3} \dot{\varepsilon}_{s}^{\mathrm{eq}}\right)^{m-1}$

where the viscoplastic consistency, $K_{v}$, and the strain-rate

Table 1 Volume-averaged conservation equations

\begin{tabular}{ll}
\hline Mass cons. & $\frac{\partial g_{i} \rho_{i}}{\partial t}+\nabla \cdot\left(g_{i} \rho_{i} \mathbf{v}_{i}\right)=\mp M_{l s}$ \\
Momentum cons. & $\frac{\partial g_{i} \rho_{\mathbf{v}_{i}}}{\partial t}+\nabla \cdot\left(g_{i} \rho_{i} \mathbf{v}_{i} \mathbf{v}_{i}\right)=-g_{i} \nabla p+\nabla \cdot g_{i} \tau_{i}^{\text {eff }} \mp \mathbf{U}_{l s}$ \\
Species cons. & $\frac{\partial \rho_{i} \rho_{i} c_{i}}{\partial t}+\nabla \cdot\left(g_{i} \rho_{i} \mathbf{v}_{i} c_{i}\right)=\mp C_{l s}$ \\
Enthalpy cons. & $\frac{\partial g_{i} \rho_{i} h_{i}}{\partial t}+\nabla \cdot\left(g_{i} \rho_{i} \mathbf{v}_{i} h_{i}\right)=-\nabla \cdot \mathbf{q}_{i} \mp H_{l s}$ \\
Grain transport & $\frac{\partial n}{\partial t}+\nabla \cdot\left(\mathbf{v}_{s} n\right)=0$
\end{tabular}

Grain transport

$\frac{\partial n}{\partial t}+\nabla \cdot\left(\mathbf{v}_{s} n\right)=0$ 
sensitivity, $m$, are material-dependent quantities. In the present work, they are defined as $6.31 \times 10^{6} \mathrm{~Pa} \cdot \mathrm{s}$ and 0.213 , respectively. The equivalent strain rate is in turn given by

$\dot{\varepsilon}_{s}^{\mathrm{eq}}=\sqrt{\frac{2}{A}\left(\dot{\varepsilon}_{s}: \dot{\varepsilon}_{s}\right)-\left(\frac{2}{3 A}-\frac{1}{9 B}\right) \operatorname{tr}\left(\dot{\varepsilon}_{s}\right)^{2}}$.

Coupling between the phases is achieved through the single pressure field, $p$, and the different interphase exchange terms $M_{\ell s}, \mathbf{U}_{\ell s}, C_{\ell s}$, and $H^{*}$ which are presented in Table 2. The quantities $v_{r}, S_{\ell s}, \Phi_{\text {imp }}$ and $K_{\ell s}$ are the crystal growth velocity, specific surface area, impingement factor and momentum exchange coefficient respectively. They have been described in detail in companion papers [7, 8]. So the readers are requested to refer to those papers for further details. The enthalpy exchange term is used to satisfy the condition of thermal equilibrium between phases (with $\left.h_{c}=10^{9} \mathrm{~W} / \mathrm{m}^{2} / \mathrm{K}\right)$. Lastly, the effective thermal diffusivity for enthalpy in Eq. (16) is equal to $2.49 \times 10^{-5} \mathrm{~m}^{2} / \mathrm{s}$ for the liquid and $7.29 \times 10^{-5} \mathrm{~m}^{2} / \mathrm{s}$ for the solid.

\section{Results and Discussion}

Twin-roll continuous casting has been the subject of extensive research due to the increasing interest from industry. It is also an ideal scenario for demonstrating the applicability and robustness of the proposed splitting algorithm which has been developed to account for the viscoplastic regime in an OpenFOAM-based numerical model originally used to address exclusively solidification and transport of equiaxed crystals.

The schematic diagram of the geometry replicated here is shown in Fig. 1, whereas the boundary conditions used in the simulation are presented in Table 3. The presented simulation results are for an inoculated $\mathrm{Al}-4 \mathrm{wt} \% \mathrm{Cu}$ alloy $(\cong 2.5 \mathrm{~mol} \%)$. Melt is fed at the inlet of the domain, where a fixed pressure field is specified $\left(p=10^{5} \mathrm{~Pa}\right)$. Dirichlet boundary conditions are imposed at the inlet to solid fraction ( $g_{s}=10^{-3}$, i.e., a practically zero amount), liquid and solid species mass fractions $\left(c_{\ell}=2.5 \mathrm{~mol} \%\right.$ and $c_{s}=0.36 \mathrm{~mol} \%$ according to phase diagram) and initial crystal diameter $(d=5 \mu \mathrm{m})$. A heat flux boundary condition is imposed to the roll and strip surfaces, with $T_{\infty}=300 \mathrm{~K}$. The solid and liquid heat capacities have been defined as $c_{P, s}=766 \mathrm{~J} / \mathrm{K}$ and $c_{P, \ell}=1179 \mathrm{~J} / \mathrm{K}$, whereas the solid and liquid thermal conductivities have been defined as $\lambda_{s}=153 \mathrm{~W} / \mathrm{m} / \mathrm{K}$ and $\lambda_{\ell}=77 \mathrm{~W} / \mathrm{m} / \mathrm{K}$. The remaining fields not presented in Table 3 are set with homogeneous Neumann boundary conditions.

The steady-state qualitative outcome of the twin-roll continuous casting simulation is presented below. Figure 2 illustrates the steady-state results for the solid fraction (top), temperature (middle) and effective solid viscosity (bottom, with logarithmic scale). The black line signals the interface at which the viscoplastic model is triggered. It can be seen that the melt cools from $920 \mathrm{~K}$ at the inlet of the domain to below $700 \mathrm{~K}$ right after the roll nip. Below the eutectic temperature (around $825 \mathrm{~K}$ ), no solidification source term is allowed in the simulation. Therefore, the last portion of the process has no relevant dynamics to highlight, with the strip leaving the outlet with a solid fraction close to $100 \%$.

From the bottom snapshot, one can notice the huge increase in viscosity as the solidification process materializes. At the interface where the viscoplastic regime is employed, the viscosity jumps several orders of magnitude, which increases considerably the stiffness of the problem. Looking to the solid fraction snapshot, one can notice a region with almost zero solid fraction right upstream of the region where the two nearly fully solidified shells meet, along the centerline of the domain. This phenomenon can be explained by the development of a back flow depicted in Fig. 3.

As the partly solidified shells are pressed together, material consisting of segregated melt and also some solid is squeezed out against the casting direction. The corresponding solid thus moves into an area where the conditions change from solidification to melting, and therefore the solid gradually disappears. Also, the crystals that are formed further upstream melt by moving into this area of highly segregated liquid. A flow reversal in the vicinity of the roll nip was also reported in Ref. [4]. Similar to the

Table 2 Exchange terms and other closure laws for the conservation equations

\begin{tabular}{lll}
\hline Mass transfer & $M_{\ell s}=v_{r} S_{\ell s} \rho_{s} \Phi_{\text {imp }}$ & \\
Momentum transfer & $\mathbf{U}_{\ell s}=K_{\ell s}\left(\mathbf{v}_{\ell}-\mathbf{v}_{s}\right)$ & $(12)$ \\
Species transfer & $C_{\ell s}=c_{s}^{*} M_{\ell s}$ \\
Enthalpy transfer & $H^{*}=h_{c}\left(T_{\ell}-T_{s}\right)$ \\
Heat flux & $\mathbf{q}_{i}=\alpha_{i} \nabla h_{i}$ \\
\hline
\end{tabular}


Fig. 1 Schematic

representation of the twin-roll continuous casting process

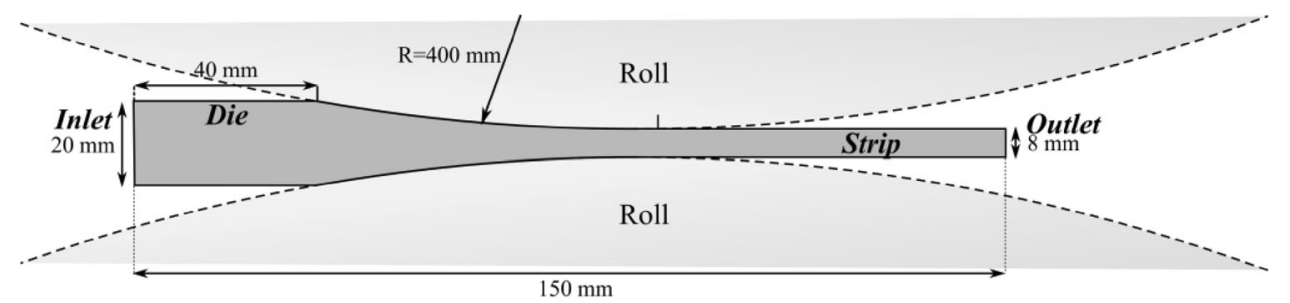

Table 3 Boundary conditions for velocities and enthalpy/temperature fields

\begin{tabular}{llll}
\hline & Solid velocity & Liq. velocity & Enthalpy/temperature \\
\hline Inlet & Pressure inlet & Pressure inlet & $925 \mathrm{~K}$ \\
Die & Slip & No slip & $925 \mathrm{~K}$ \\
Roll & Slip & $0.085 \mathrm{rad} / \mathrm{s}$ & Heat flux $\left(\mathrm{HTC}=11 \mathrm{~kW} / \mathrm{m}^{2} / \mathrm{K}\right)$ \\
Strip & Slip & $0.034 \mathrm{~m} / \mathrm{s}$ & Heat flux $\left(\mathrm{HTC}=2 \mathrm{~W} / \mathrm{m}^{2} / \mathrm{K}\right)$ \\
Outlet & $0.034 \mathrm{~m} / \mathrm{s}$ & $0.034 \mathrm{~m} / \mathrm{s}$ & Zero gradient \\
\hline
\end{tabular}

Fig. 2 Steady-state results for the solid fraction (top) and liquid temperature (middle) and logarithmic scale) effective viscosity (bottom, with
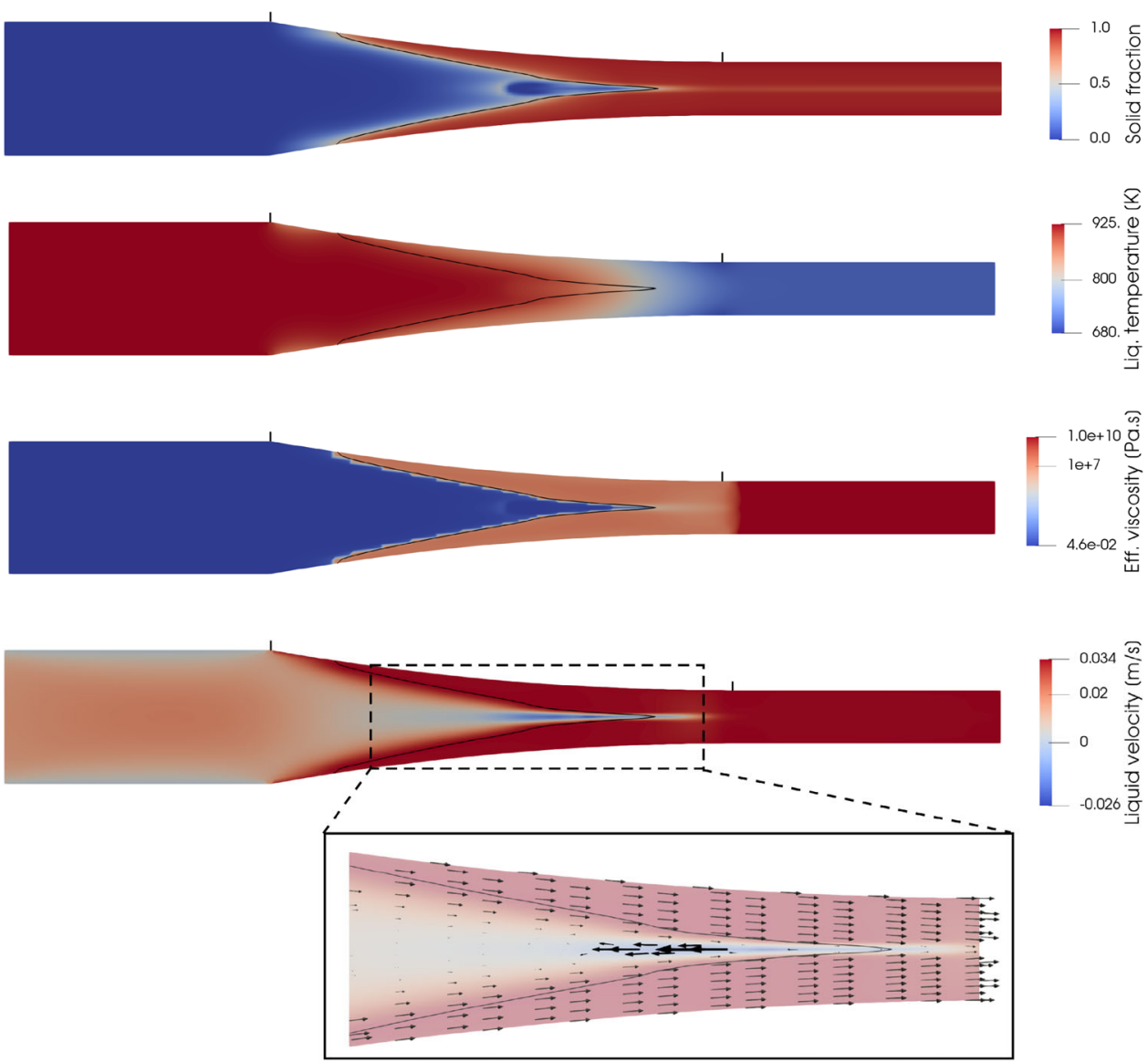

Fig. 4 Macrosegregation pattern along domain

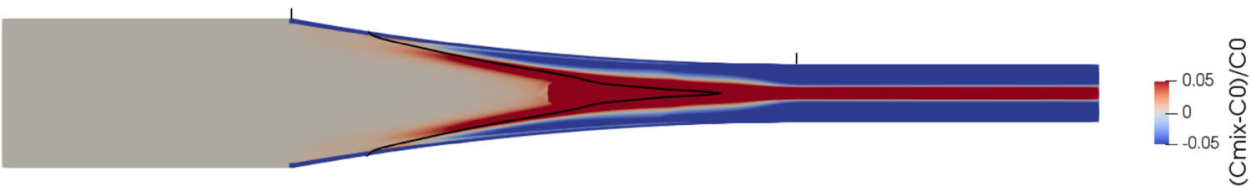


present work, the authors had also considered viscoplasticity of the semi-solid. However, they did not account for segregation and thus melting was not predicted.

The formation of macrosegregation is a severe problem in nearly all continuous casting processes, and so in twinroll casting. Figure 4 shows the deviation of local alloy composition from the nominal value, $c_{0}$, in terms of the relative mixture concentration, $\left(c_{\text {mix }}-c_{0}\right) / c_{0}$. The origin of the macrosegregation pattern illustrated if Fig. 4 is mainly due to the deformation of the viscoplastic mush, which means that segregated melt can be squeezed out of the coherent structure, inducing negative segregation in the region subjected to deformation. Accordingly, it can be seen in Fig. 4 that during hot rolling, negative segregation starts to develop near the surfaces of the rolls and then extends to the end of the strand as the casting proceeds. On the other hand, as a result of the interstitial liquid being expelled from the deformed mush, it travels to adjacent inner regions, causing positive segregation there. Furthermore, due to the reverse flow-which consists of highly segregated melt-strong positive macrosegregation can be found upstream of the kissing point.

\section{Conclusion}

The need for high-quality strips during twin-roll continuous casting makes it imperative for the research community to achieve a better understanding of the physical mechanisms underlying this material process technique. The present model paves the way for such an accomplishment, since it makes it much easier to analyze the influence of different casting parameters in order to build an optimized operation plan. The presented results show that when the partly solidified, viscoplastic shells from both rolls meet at the kissing point, material is squeezed out against casting direction, leading to a local solutally enriched area where crystals melt. The described phenomenon contributes to the formation of a strong centerline macrosegregation.

Acknowledgements Open access funding was provided by Montanuniversität Leoben. This work was financially supported by the FWF Austrian Science Fund (P28785-N34) which the authors gratefully acknowledge.

Open Access This article is distributed under the terms of the Creative Commons Attribution 4.0 International License ( http://creativecommons.org/licenses/by/4.0/), which permits unrestricted use, distribution, and reproduction in any medium, provided you give appropriate credit to the original author(s) and the source, provide a link to the Creative Commons license, and indicate if changes were made.

\section{References}

1. Bondarenko S, Stolbchenko M, Schaper M, and Grydin O, Mater Res 21 (2018) e20171098:1.

2. Matsushita T, Nakayama K, Fukase H, and Osada S, IHI Eng Rev 42 (2009) 1.

3. Mortensen D, Fjaer H G, Lindholm D, Karhausen K F, and Kvalevag J S, TMS-Light Met (2015) 1243-1247.

4. Lee Y S, Kim H W, Cho J H, and Chun S H, Met Mater Int 23 (2017) 923.

5. Sahoo S, Kumar A, Dhindaw B K, and Ghosh S, Met Mater Trans B 43 (2012) 915.

6. Nguyen T G, Favier D, and Suery M, Int J Plast 10 (1994) 663.

7. Ludwig A, and Wu M, Met Mater Trans A 33 (2002) 3673.

8. Wu M, and Ludwig A, Acta Mater 57 (2009) 5621.

9. Fachinotti V D, Le Corre S, Triolet N, Bobadilla M, and Bellet M, Int J Numer Methods Eng 67 (2006) 1341.

10. Ishii M, Thermo-Fluid Dynamic Theory of Two-Phase Flow, Eyrolles, Paris (1975).

11. Olmedilla A, Založnik M, and Combeau H, EPJ Web Conference, 140 (2017). 